(2)

\title{
Persistent high fever and systemic inflammation induced by percutaneous coronary intervention-related periaortitis
}

This article was published in the following Dove Press journal:

Vascular Health and Risk Management

7 December 2009

Number of times this article has been viewed

\author{
Takamori Kakino \\ Tomohiro Nakayama \\ Hideo Yamamoto \\ Department of Cardiology and \\ Radiology, Kyushu Kosei-Nenkin \\ Hospital, Kitakyushi City, Japan
}

Correspondence: Hideo Yamamoto Department of Cardiology, Kyushu Kosei-Nenkin Hospital, I-8-I Kishinoura, Yahata-Nishiku, Kitakyushu City 806850I, Japan Email hideo.yamamoto@qkn-hosp.jp

\begin{abstract}
After coronary catheterization, fever sometimes occurs, usually transiently. We report a very rare case that suggests persistent high fever and systemic inflammation might be caused by periaortitis induced by coronary catheterization including percutaneous coronary intervention.
\end{abstract}

Keywords: periaortitis, percutaneous coronary intervention, fever, computed tomography

\section{Case report}

A 79-year-old man was admitted to our hospital because of a two-month history of effort angina. He had smoked 70 cigarettes daily for about 50 years until seven years ago and had been treated for hypercholesterolemia for more than 10 years. He felt perfectly well until the occurrence of effort angina. Five days before admission, multidetector-row computed tomography (MDCT) of the heart showed severe stenosis of the right coronary artery and no contrast enhancement of the thoracic aorta. Coronary angiography from the right femoral artery approach showed $90 \%$ stenosis of the right coronary artery segment 2, in which a drug-eluting stent (DES; sirolimus-eluting stent) was placed. Twelve hours after placement of the DES, he developed a $39{ }^{\circ} \mathrm{C}$ fever with chills and rigor. The fever continued, but physical examinations were normal. He had no arthritis or eruptions, including so-called "blue toes" in the extremities. Repeated chest X-rays, electrocardiogram and echocardiography were normal. Laboratory examinations showed raised C-reactive protein (CRP; normal value $<0.25 \mathrm{mg} / \mathrm{dL}$ ) of $20.1 \mathrm{mg} / \mathrm{dL}$ from $0.3 \mathrm{mg} / \mathrm{dL}$ five days before admission and white blood cell count was $8600 / \mu \mathrm{L}$ without eosinophilia. Plasma levels of cytokines such as interleukin-6 (IL-6) (normal value $<4.0 \mathrm{pg} / \mathrm{mL}$ ), IL-8 (normal value $<2.0 \mathrm{pg} / \mathrm{mL}$ ) and tumor necrosis factor- $\alpha$ (TNF- $\alpha$ ) (normal value $0.6-2.8 \mathrm{pg} / \mathrm{mL}$ ) were significantly increased $(25.7 \mathrm{pg} / \mathrm{mL}$, $12.9 \mathrm{pg} / \mathrm{mL}, 3.4 \mathrm{pg} / \mathrm{mL}$, respectively). Antinuclear antibody (160 times, normal value $<40$ times) and rheumatoid factor (640 times, normal value $<40$ times) were positive. Repeated cultures of blood, urine, and sputum were all negative.

On the 12th day, a contrast-enhanced computed tomography (CT) was performed and a $3.3 \mathrm{~cm}$ infrarenal abdominal aortic aneurysm (AAA) was found, of which the maximum wall thickness was $8 \mathrm{~mm}$ with periaortic dirty fat signs. The periaortic contrast-enhancement from the ascending thoracic aorta including the branches of the aortic arch to the common iliac arteries, noted abnormalities which were especially pronounced from AAA to the common iliac arteries (Figure 1). The wall of aorta showed advanced atherosclerosis. The CT images suggested the periaortitis such as 

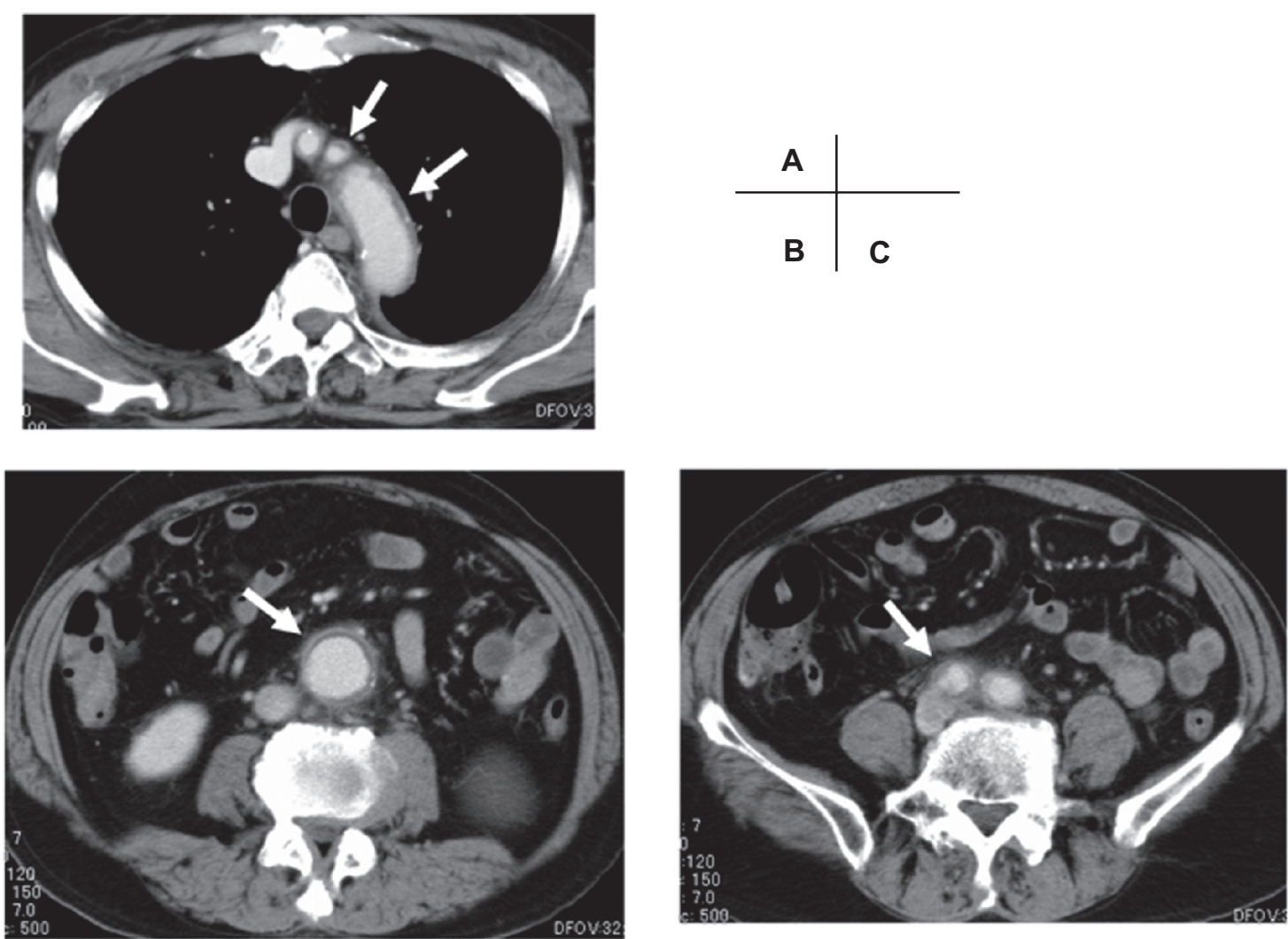

Figure I Contrast computed tomography showed the periaortic enhancement and dirty fat sign (white arrow), at the level of aortic arch and its branches A) abdominal aortic aneurysm, B) and common iliac arteries $\mathbf{C})$.

inflammatory abdominal aortic aneurysm (IAAA). He was treated with predonisolone $40 \mathrm{mg}$ per day from the 14th day, resulting in complete resolution of fever over the next day. Additionally, CRP fell to $0.05 \mathrm{mg} / \mathrm{dL}$ and plasma levels of cytokines recovered to the normal range on the 28th day. The ANA and RA factor became negative. The CT on the 38th day showed that the periaortic dirty fat sign and contrastenhancement disappeared (Figure 2). Predonisolone was withdrawn successfully one year after the treatment.

\section{Discussion}

After coronary catheterization, fever sometimes occurs usually transiently. The etiologies of fever are thought to be local and systemic infection, allergic reactions against contrast material, inflammatory reactions against hematoma, and embolic reactions such as blue toe syndrome. We thought the persistent high fever and systemic inflammation of this patient might be caused by an inflammatory response in the aortic wall, because the $\mathrm{CT}$ showed a finding of periaortitis and after predonisolone therapy the persistent high fever decreased, the increased CRP and cytokines recovered to the normal range, and the CT findings disappeared. It is known that the increase in systemic markers of inflammation including cytokines after percutaneous coronary intervention is frequently attributed to the inflammatory stimulus associated with coronary artery injury during balloon inflation and stent implantation ${ }^{1}$ and that, even after diagnostic coronary angiography, a systemic inflammatory response occurs in patients with stable effort angina. ${ }^{2}$ However, these kinds of systemic inflammations occur transiently without the persistent high fever.

The CT findings of periaortitis in this patient are similar to those of IAAA, which is one of the three forms of chronic periaortitis: 1) IAAA, 2) idiopathic retroperitoneal fibrosis, and 3) perianeurysmal retroperitoneal fibrosis which is a combination of the two disorders. ${ }^{3}$ The three forms of chronic periaortitis have similar histopathological characteristics, including adventitial and periadventitial inflammation, medial thinning and advanced atherosclerosis, and thus represent different manifestations of the same disease. The definitive diagnostic test of the chronic periaortitis is contrastenhanced CT scanning or gadolinium-enhanced magnetic resonance imaging. These demonstrate the periaortic soft tissue mass as a rind of abnormal tissue around the aorta, 

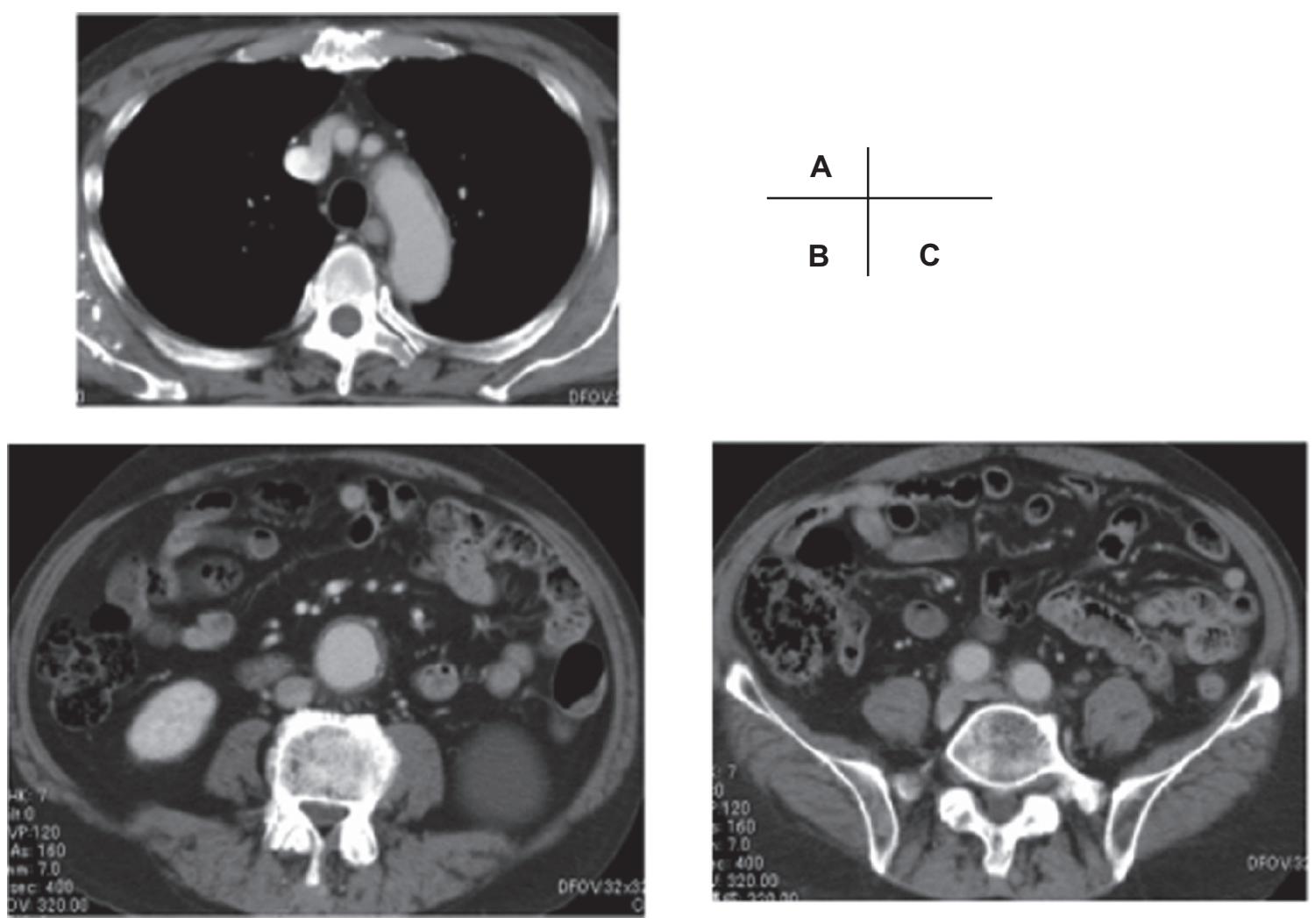

Figure 2 Contrast computed tomography three weeks after steroid therapy at the level of aortic arch and its branches A) abdominal aortic aneurysm, B) and common iliac arteries $\mathbf{C})$.

with a varying extent of spread, and the aorta is aneurysmal in IAAA. ${ }^{3}$ Iino and colleagues reported sensitivity of $83.3 \%$, specificity of $99.7 \%$, and accuracy of $93.7 \%$ for a diagnosis of IAAA using CT scanning. ${ }^{4}$ We do not have any histopathological evidence of periaortitis, but the CT images of this patient showed the periaortic contrast enhancement from the ascending thoracic aorta including the branches of the aortic arch to the common iliac arteries, which was especially pronounced from AAA to the common iliac arteries and this periaortic contrast enhancement might be the major feature that distinguishes inflammatory from atherosclerotic AAA. Although the etiology of IAAA is unknown, IAAA is thought to be a consequence of local inflammatory reaction to antigens such as oxidized low-density lipoproteins (LDL) and ceroid (a lipoproteic polymer that results from LDL oxidation), which are often found in the advanced atherosclerotic plaques. This causes antigen-antibody reactions concentrated in the media and adventitia, leading to inflammation and fibrotic proliferation around the aorta. On the other hand, patients with IAAA often have systemic symptoms, raised acute phase reactants, and positive autoantibodies and the inflammatory process not only involves the abdominal aorta but also the thoracic aorta and their branches ${ }^{3}$ as noted with this patient. The evaluation of chronic periaortitis by positron emission tomography showed a vascular inflammatory process mainly localized to the abdominal aorta and/or to the common iliac arteries, but the involvement of the thoracic aorta and its branches has been observed in $43 \%$ of chronic periaortitis patients. ${ }^{5}$ These suggest that IAAA could be a systemic autoimmune disease as seen in systemic vasculitis such as aortitis syndrome. ${ }^{3,5}$ In this patient, we presume that the atherosclerotic plaques of the aorta, especially in the infrarenal AAA could be stimulated locally by the catheters during the cardiac catheterization, causing activation of the antigen-antibody reaction from thoracic to abdominal aorta and their branches as described above, and resulted in acute systemic inflammatory responses including high fever.

In this patient before admission, systemic inflammation was not seen and the MDCT of the heart showed no signs of periaortitis at the level of the thoracic aorta, however an abdominal aortic CT was not performed. Therefore, it is not clear whether the AAA in this patient might be an asymptomatic early phase of IAAA without retroperitoneal fibrosis or only an advanced atherosclerotic AAA. Because it is known 
that the inflammatory variant is seen in $5 \%$ to $10 \%$ of all cases of AAA, IAAA is not uncommon ${ }^{6}$ and pathological findings support that periaortitis can precede idiopathic retroperitoneal fibrosis. ${ }^{3}$ Asymptomatic early phase of IAAA might be easily activated to periaortitis by catheter stimulation and also by nonspecific inflammatory reactions such as a cytokines storm triggered by stent implantation, which was seen in this case. Therefore, careful observation of a patient is important because of the possibility of early phase of IAAA.

There are some reports about secondary IAAA induced by some medications (methysergide, methyldopa, amphetamines, cocaine, and beta-blockers), malignant diseases, infections, radiotherapy, and surgery (lymphadenectomy, colectomy, hysterectomy, aortic aneurysmectomy), ${ }^{3}$ none of which were related to this patient. There had not been any reports about secondary IAAA related to endovascular treatment, but recently, there are some reports that endovascular therapy of AAA also had a severe and rare complication of IAAA. ${ }^{7,8}$ To our knowledge, this patient might be the first case of secondary periaortitis induced by the coronary catheterization and percutaneous coronary intervention.

In conclusion, when persistent high fever and systemic inflammation occur after catheterization and usual etiologies of fever are not found, we should consider periaortitis, especially in patients with advanced atherosclerotic AAA.

\section{Disclosures}

The authors report no conflicts of interest in this work.

\section{References}

1. Saleh N, Svane B, Jensen J, et al. Stent implantation, but not pathogen burden, is associated with plasma C-reactive protein and interleukin-6 levels after percutaneous coronary intervention in patients with stable angina pectoris. Am Heart J. 2005;149:876-882.

2. Goldberg A, Zinder O, Zdorovyak A, et al. Diagnostic coronary angiography induces a systemic inflammatory response in patients with stable angina. Am Heart J. 2003;146:819-823.

3. Vaglio A, Salvarani C, Buzio C. Retroperitoneal fibrosis. Lancet. 2006;367:241-251.

4. Iino M, Kuribayashi S, Imakita S, et al. Sensitivity and specificity of CT in the diagnosis of inflammatory abdominal aortic aneurysm. J Comput Assist Tomogr. 2002;26:1006-1012.

5. Salvarani C, Pipitone N, Versari A, et al. Positron emission tomography (PET): Evaluation of chronic periaortitis. Arthritis Rheum. 2005;53:298-303.

6. Hellmann DB, David JG, Julie AF. Inflammatory abdominal aortic aneurysm. JAMA. 2007;297:395-400.

7. Brouw LW, van Weerelt CTC, van Guldener C, et al. Non invasive treatment of peri-aortic inflammation after endovascular graft. Eur $J$ Vasc Endovasc Surg. 2007;34:179-181.

8. Simons PC, van Overhagen H, Bruijninckx CM, et al. Periaortitis with ureteral obstruction after endovascular repair of an abdominal aortic aneurysm. AJR Am J Roentgenol. 2002;179:118-120.
Vascular Health and Risk Management

\section{Publish your work in this journal}

Vascular Health and Risk Management is an international, peerreviewed journal of therapeutics and risk management, focusing on concise rapid reporting of clinical studies on the processes involved in the maintenance of vascular health; the monitoring, prevention and treatment of vascular disease and its sequelae; and the involvement of

\section{Dovepress}

metabolic disorders, particularly diabetes. This journal is indexed on PubMed Central and MedLine. The manuscript management system is completely online and includes a very quick and fair peer-review system, which is all easy to use. Visit http://www.dovepress.com/ testimonials.php to read real quotes from published authors. 